\title{
Measurements of Reynolds stress in a Wind Driven Lagoonal Estuary
}

\author{
ANTHONY CLYDE WHIPPLE
}

A thesis submitted to the faculty of the University of North Carolina at Chapel Hill in partial fulfillment of the requirements for the degree of Master of Science in the Department of Marine Sciences.

Chapel Hill

2007

Approved by:

Richard Luettich

Harvey Seim

John Bane 


\begin{abstract}
ANTHONY WHIPPLE: Measurements of Reynolds stress in a Wind Driven Lagoonal Estuary (Under the direction of Richard Luettich)
\end{abstract}

ADCPs have been used to measure Reynolds stresses in tidally dominated environments where wave action was minimal. In this thesis we examine observations from a microtidal estuary where the effects of wind stress and surface waves dominate the velocity variance. Reynolds stress measurements in this setting require a technique for addressing surface gravity wave contamination. We present here a method of reducing the effect of wave motion on Reynolds stresses by subtracting coincident observations along the axis of the ADCP beam. Linear wave theory is used to account for the attenuation of wave orbital velocities with depth. Using this method, Reynolds stress values are brought in line with those predicted by drag laws at the surface and bottom. Coincident density profile measurements are used with the ADCP data to compute gradient Richardson numbers throughout the water column. Enhanced Reynolds stresses appear to correspond to Richardson numbers less than one. 


\section{Table of Contents}

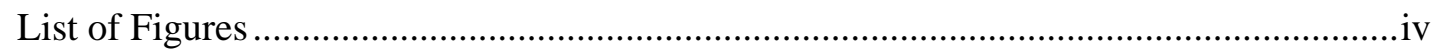

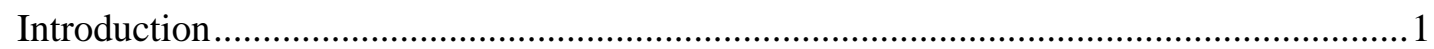

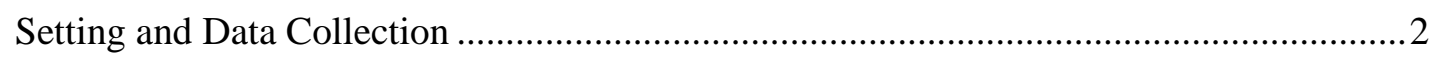

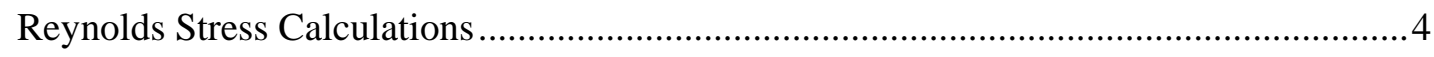

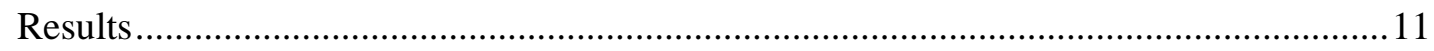

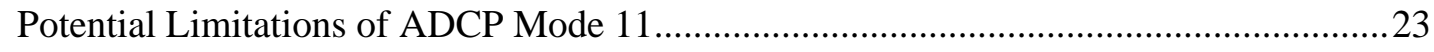

Effect of the Vertical Separation Distance in the Wave - Turbulence Separation.............25

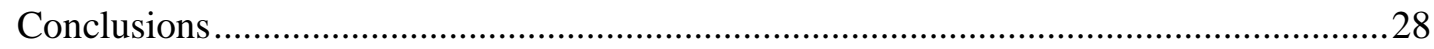

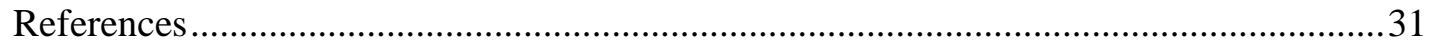




\section{List of Figures}

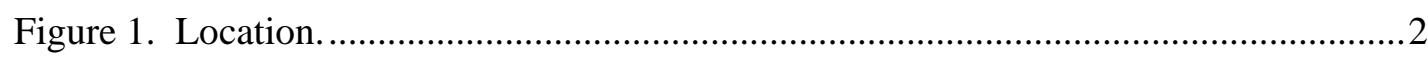

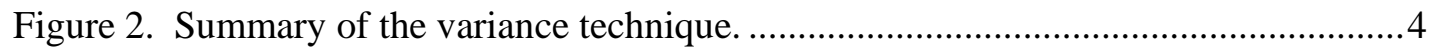

Figure 3. Several typical variance curves and curve fits. .............................................

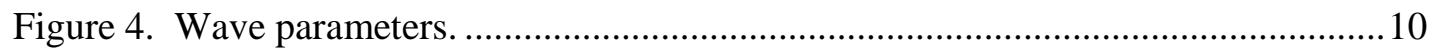

Figure 5. Spectra of along beam velocity and Reynolds stress...................................... 12

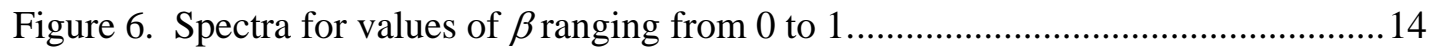

Figure 7. Drag law compared to Reynolds stress. ...................................................... 15

Figure 8. Error due to the interaction of wave motion with instrument tilt ......................17

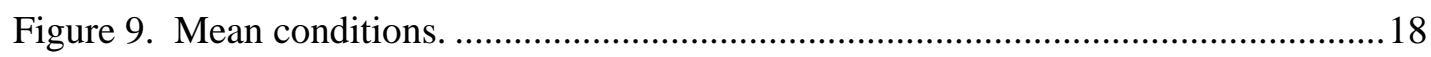

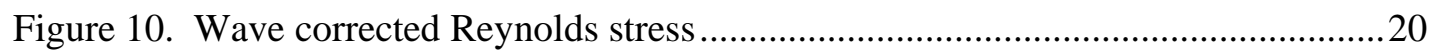

Figure 11 Richardson number verses Reynolds stress magnitude.................................21

Figure 12 Reynolds stress by standard deviation of Reynolds stress .............................23

Figure 13 The number of good data values …............................................................. 24

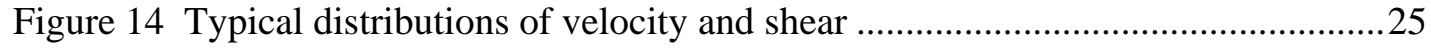

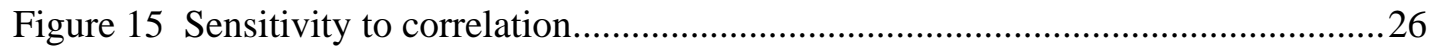

Figure 16 Reynolds stress spectra for three separation distances ...................................27 


\section{Introduction}

The use of ADCP's to measure Reynolds stresses has been rapidly gaining popularity. One of the most promising procedures, called the "variance technique”, was described by Stacey et al. (1999). Early work calculating Reynolds stresses using the variance technique with a 4-beam ADCP was done in controlled environments that were tidally dominated with minimal wave action (Stacey et al, 1999; Lu and Lueck, 1999).

In this paper we examine observations from a microtidal estuary where the effects of wind and surface waves dominate the velocity variance. Using the variance technique in such an environment, surface waves can generate errors in estimates of Reynolds stresses due to non-linear wave motion or instrument tilt (Trowbridge, 1998; Rippeth et al., 2003). Empirically we have observed that, in the presence of waves, Reynolds stresses using the variance technique can be an order of magnitude or more larger then predicted by drag laws at the surface and bottom. Wave-Reynolds stress separations have been performed using other instrumentation, for example simultaneous pressure and LDV velocity measurements (Agrawal and Aubrey, 1992) and two horizontally arrayed velocity sensors, (Trowbridge, 1998). However, none have been presented using a single ADCP.

This paper presents an along beam differencing method for wave-Reynolds stress separation for use with a single 4-beam ADCP. Profiles of Reynolds stress and gradient Richardson number are computed for a two-week period near the mouth of the Neuse River Estuary. Water velocities were obtained using a bottom mounted, upward looking, $1200 \mathrm{kHz}$ ADCP using the shallow water, low energy mode 11 feature. Water column 
density structure was determined using an autonomous vertical profiler (ReynoldsFleming et al., 2002).

\section{Setting and Data Collection}

The Neuse River Estuary is a part of the Albemarle-Pamlico Estuarine system, which is the largest lagoonal estuary in the United States. As a result of the limited exchange with the open ocean, the tide signal is negligible and the predominant forcings are fresh water runoff, wind stress, and a $13.2 \mathrm{hr}$ seiche (Luettich et al., 2000, 2002).

The lower Neuse River Estuary flows from the southwest toward the northeast (figure 1). Exposure to the northeast is toward the main body of Pamlico Sound. The prevailing wind direction is from the southwest during the summer and from the northeast during the winter, with the north-easterlies being typically stronger. Water velocities in the estuary are usually less than $20 \mathrm{~cm} / \mathrm{s}$ and the most energetic response is due either to direct wind forcing or a 13.2-hour seiche (Luettich et al., 2000, 2002; Reynolds-Fleming

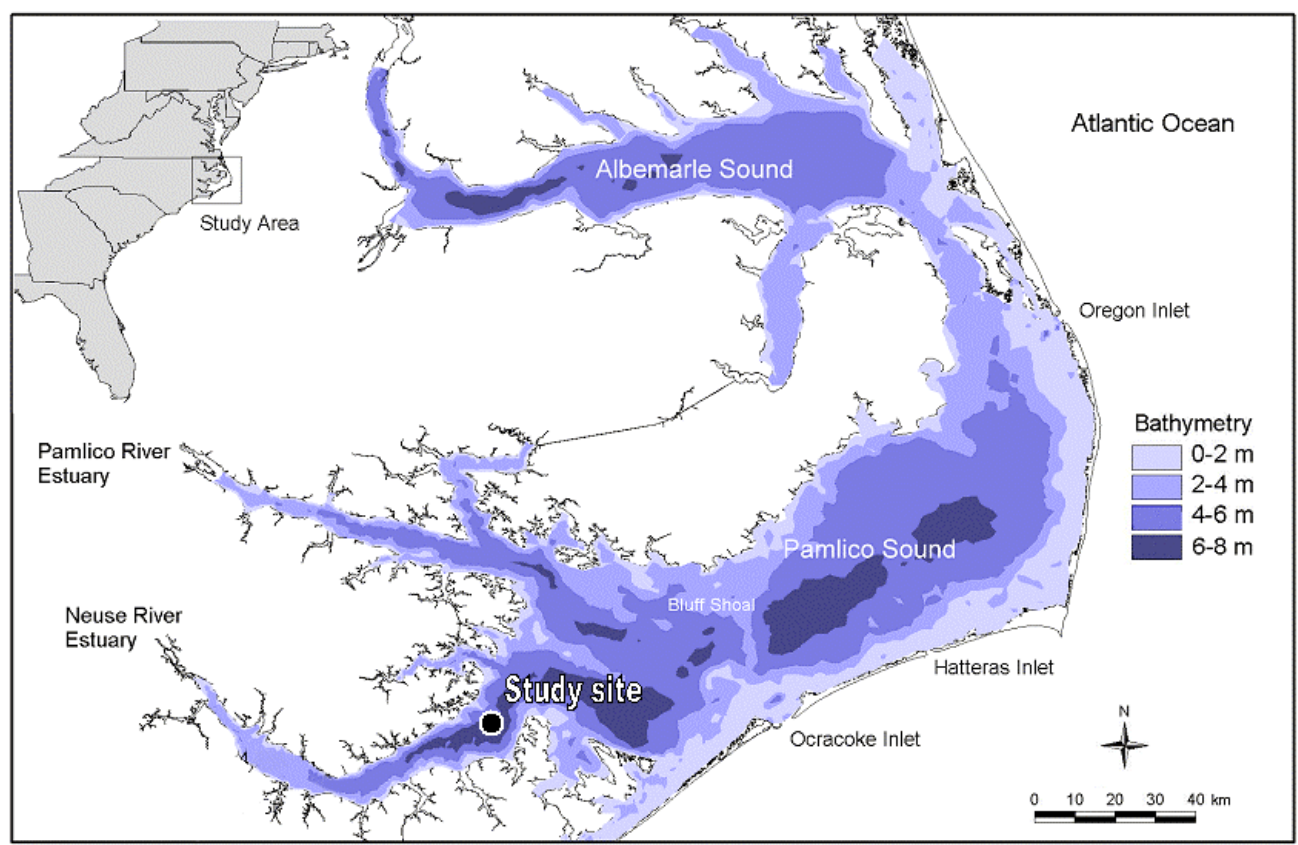

Figure 1. Location of the study site in the lower Neuse River and the adjacent Pamlico Sound, North Carolina, USA. 
et al., 2004). Stratification ranges from completely mixed in the vertical, to a change in salinity of 10 psu over the depth (Luettich et al., 2000).

Data were collected with an RDI $1200 \mathrm{kHz}$ 4-beam ADCP. The ADCP was mounted in a frame in an upward looking orientation and placed on the bottom of the Neuse River Estuary in approximately 7 meters of water. A bottom-mounted pressure gauge was located about 10 meters from the ADCP. Data from this pressure gauge was used to mask the water height in the ADCP's data stream. The ADCP was deployed from October 30 -November 15, 2003, yielding 15.5 days of data. Ensembles were taken every 30 minutes with a vertical resolution of $10 \mathrm{~cm}$. The low water velocities and shallow environment allowed us to use the ADCP's low variability shallow water mode 11. The sampling scheme was to ping once per second for the first 15 minutes of each half hour. There was no averaging and each ping was saved in raw beam co-ordinates.

Great care was taken to install the ADCP as close to vertical as possible. The maximum tilt angle for our deployment was 1 degree as determined by the ADCP's internal pitch and roll sensors. Instrument tilt will impact the estimated Reynolds stresses in two distinct ways. First, in the absence of wave motion the Reynolds stresses will contain errors due to the presence of horizontal turbulent velocities in the measured vertical turbulent velocities. In our case the tilt should result in less than a 10 percent error in the Reynolds stress estimates and consequently this was not considered to be significant (Lu and Lueck, 1999). Second, in the presence of wave motion, the tilt error will cause a covariance of the wave velocity components which contaminates the Reynolds stress estimates (Rippeth et al., 2003). As shown below this provides a much more significant source of error to Reynolds stress estimates using the variance technique unless the wave motions are first removed from the velocity signal. 
Also located about 10 meters away from the ADCP was a floating platform based autonomous profiling system (Reynolds-Fleming et al., 2002). Every 30 minutes this device profiled the water column ( $4 \mathrm{~cm}$ vertical resolution) using a YSI 6600 Sonde collecting salinity, temperature, dissolved oxygen, turbidity and chlorophyll concentration data.

\section{Reynolds Stress Calculations}

Figure 2 summarizes the variance technique for calculating Reynolds stresses using an upward looking ADCP. Crucial to this technique is the subtraction of the variance of opposing beams. As a consequence of this subtraction (and with a perfectly vertical instrument), the variance resulting from wave motions that adhere to linear wave theory should cancel out. This can be demonstrated by assuming an orthogonal set of sinusoidal wave velocity components and computing the along beam wave velocities:

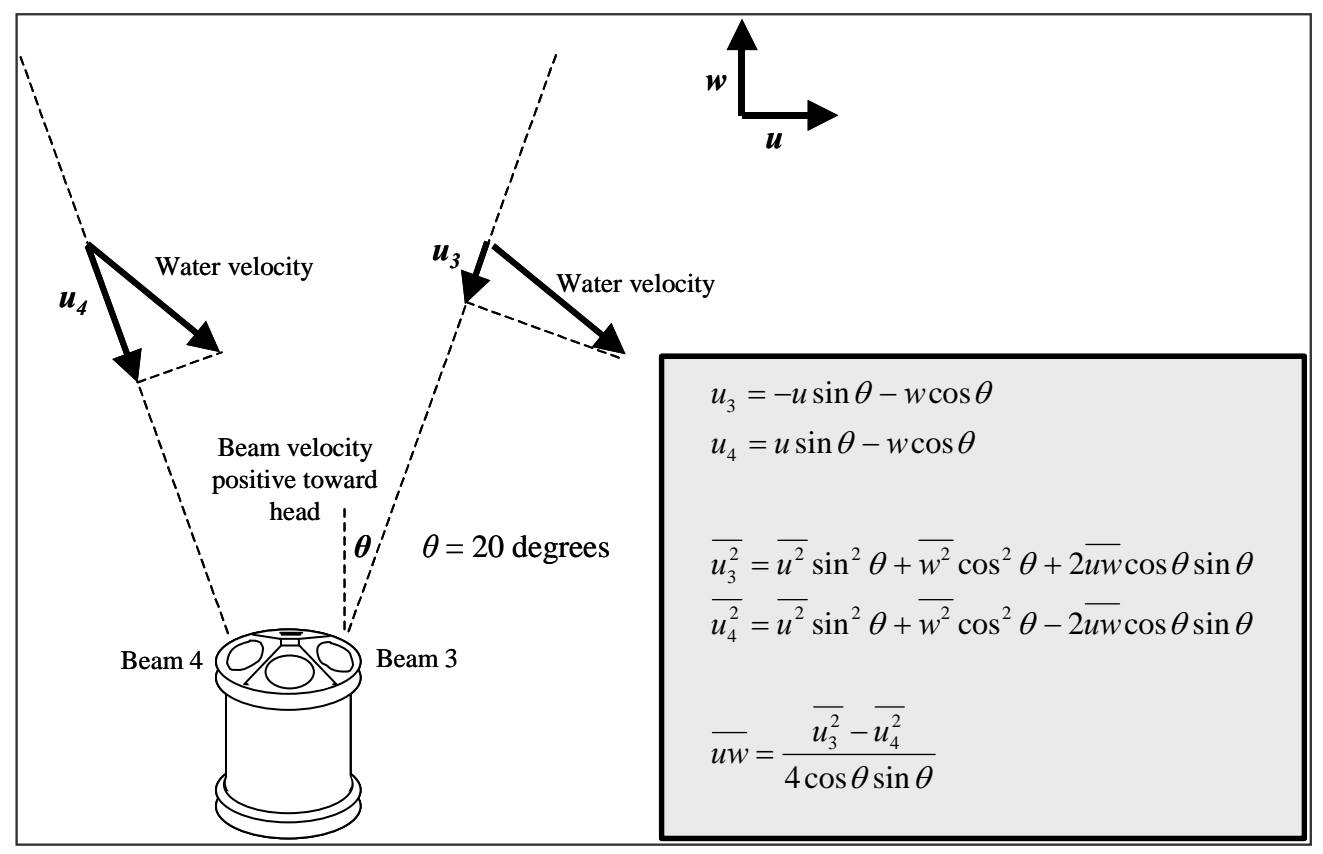

Figure 2. Summary of the variance technique for calculating Reynolds stresses for an upward looking ADCP. Variables $u$ and $w$ are the de-meaned horizontal and vertical velocity components in the plane defined by beams 3 and 4 . The de-meaned along beam velocity components are given as $u_{3}$ and $u_{4}$ and are positive toward the Transducer head. Overbars indicate time averaging. 


$$
\begin{aligned}
& \tilde{u}_{3}=-\tilde{u} \cos (k x-\omega t) \sin \theta-\tilde{w} \sin (k x-\omega t) \cos \theta \\
& \tilde{u}_{4}=\tilde{u} \cos (k x-\omega t) \sin \theta-\tilde{w} \sin (k x-\omega t) \cos \theta
\end{aligned}
$$

The variables $\tilde{u}_{3}$ and $\tilde{u}_{4}$ represent the velocity due to wave motion in opposing beams (e.g. beam 3 and beam 4 respectively). The variables $\tilde{u}$ and $\tilde{w}$ are the amplitudes of the horizontal and vertical motions respectively. The wave number is represented by $k$, and the frequency by $\omega$. The angle the ADCP beam makes with the vertical is $\theta$ (20 deg). The variance associated with these motions is:

$$
\begin{aligned}
& \overline{\tilde{u}_{3}^{2}}=\tilde{u}^{2} \overline{\cos ^{2}(k x-\omega t)} \sin ^{2} \theta+\tilde{w}^{2} \overline{\sin ^{2}(k x-\omega t)} \cos ^{2} \theta+2 \tilde{u} \overline{\cos (k x-\omega t) \sin (k x-\omega t)} \cos \theta \sin \theta \\
& \overline{\tilde{u}_{4}^{2}}=\tilde{u}^{2} \overline{\cos ^{2}(k x-\omega t)} \sin ^{2} \theta+\tilde{w}^{2} \overline{\sin ^{2}(k x-\omega t)} \cos ^{2} \theta-2 \tilde{u} \tilde{\cos (k x-\omega t) \sin (k x-\omega t)} \cos \theta \sin \theta
\end{aligned}
$$

where an overbar indicates an average over a suitably selected time interval. The last term in each expression time averages to zero. Since the first two terms in each expression are identical, subtracting the variances of each beam, as per the variance technique, causes them to cancel. In other words, the variance due to wave motions should be identical in each beam. Consequently, when using the variance technique (in the absence of tilt) to calculate Reynolds stresses, these wave motions should cancel out. However as wave motions depart from linear theory this may not be the case. For example, if $\tilde{u}$ and $\tilde{w}$ are not in quadrature the cross product terms in Eq. (2) no longer time average to zero. Instrument tilt will cause $\theta$ to differ between beams and consequently the first two terms in Eq. (2) would no longer subtract to zero. In a system where wave motions are the dominant source of velocity variance, small deviations from linear theory or small tilt may lead to large errors in measured Reynolds stress. Therefore it is desirable to remove as much of the wave-associated velocity variance as possible.

Trowbridge (1998) subtracted simultaneous measurements from instruments located approximately 0.2 meters above the bottom and horizontally separated by 
approximately 1 meter under the assumption that this distance was large with respect to the correlation distance of the turbulence and small with respect to the wavelength of the surface waves. This provided "a nearly wave-free estimate of the average of the turbulent shear stresses at the two sensors” (Trowbridge, 1998).

In our case, we have velocities at multiple points in the water column in the along bean direction of the ADCP. Thus, we have investigated the possibility of performing subtractions along each beam. Since the beam axis forms an angle with the vertical (20 degrees), there is both a vertical and a horizontal component to the along beam separation. To minimize the amount of turbulent energy that is removed by the subtraction, we should subtract velocities in bins that are separated by distances greater than the correlation distance of the turbulence. Also, the horizontal component of the separation must be small with respect to the wavelength of the surface waves. Finally, the expectation that the wave velocities will diminish with depth (and therefore between bins) must be accounted for.

We assume that $u_{3}$ and $u_{4}$ represent the de-meaned along beam velocities for beams 3 and 4 respectively, which form one of the vertical measurement planes of the ADCP (figure 2). A second subscript, the letter $a$ or $b$, represents one of two different positions along the axis of the beam with $a$ being farther from the transducer head than $b$. If the de-meaned velocities are partitioned into turbulent (primes) and wave (tildes) components, the velocities at level $a$ are scaled by a parameter $\beta$ and subtracted from the velocities at level $b$, the result is:

$$
\begin{aligned}
& \Delta u_{3} \equiv u_{3 b}-\beta u_{3 a}=u_{3 b}^{\prime}-\beta u_{3 a}^{\prime}+\tilde{u}_{3 b}-\beta \tilde{u}_{3 a} \\
& \Delta u_{4} \equiv u_{4 b}-\beta u_{4 a}=u_{4 b}^{\prime}-\beta u_{4 a}^{\prime}+\tilde{u}_{4 b}-\beta \tilde{u}_{4 a}
\end{aligned}
$$

The parameter $\beta$ accounts for the attenuation of the wave signal with depth and is chosen to minimize the difference between the wave terms at the two levels. Assuming that $\beta$ 
can be chosen so that the difference between these terms is zero and computing the variance of the remainder yields:

$$
\begin{aligned}
& \overline{\Delta u_{3}^{2}}=\overline{u_{3 b}^{\prime 2}}+\overline{\beta^{2} u_{3 a}^{\prime 2}}-\overline{2 \beta u_{3 b}^{\prime} u_{3 a}^{\prime}} \\
& \overline{\Delta u_{4}{ }^{2}}=\overline{{u_{4 b}^{\prime}}^{2}}+\overline{\beta^{2} u_{4 a}^{\prime 2}}-\overline{2 \beta u_{4 b}^{\prime} u_{4 a}^{\prime}}
\end{aligned}
$$

To form the expression for the Reynolds stress, the difference of the variances is taken and adjusted for the beam angle (c.f., Stacey et al, 1999).

$$
\frac{\overline{\Delta u_{3}^{2}}-\overline{\Delta u_{4}^{2}}}{4 \sin \theta \cos \theta}=\frac{\overline{u_{3 b}^{\prime 2}}-\overline{u_{4 b}^{\prime 2}}}{4 \sin \theta \cos \theta}+\frac{\beta^{2}\left(\overline{u_{3 a}^{\prime}}-\overline{u_{4 a}^{\prime}}\right)}{4 \sin \theta \cos \theta}-\frac{\overline{2 \beta u_{3 a}^{\prime} u_{3 b}^{\prime}}}{4 \sin \theta \cos \theta}+\frac{\overline{2 \beta u_{4 a}^{\prime} u_{4 b}^{\prime}}}{4 \sin \theta \cos \theta}
$$

Providing that we have chosen the distance between positions $a$ and $b$ such that the turbulent components of the velocities are uncorrelated, the time averaged product in each of the last two terms will equal zero. This leaves us with

$$
\frac{\overline{\Delta u_{3}^{2}}-\overline{\Delta u_{4}^{2}}}{4 \sin \theta \cos \theta} \approx \frac{\overline{u_{3 b}^{\prime 2}}-\overline{u_{4 b}^{\prime 2}}}{4 \sin \theta \cos \theta}+\frac{\beta^{2}\left(\overline{u_{3 a}^{\prime 2}}-\overline{u_{4 a}^{\prime 2}}\right)}{4 \sin \theta \cos \theta}=R S_{b}+\beta^{2} R S_{a}
$$

Assuming that the Reynolds stress varies slowly between $a$ and $b$ we can write

$$
\frac{\overline{\Delta u_{3}^{2}}-\overline{\Delta u_{4}^{2}}}{4 \sin \theta \cos \theta} \approx\left(1+\beta^{2}\right) R S_{a b}
$$

or

$$
R S_{a b} \approx \frac{\overline{\Delta u_{3}^{2}}-\overline{\Delta u_{4}^{2}}}{4 \sin \theta \cos \theta\left(1+\beta^{2}\right)}
$$

where $R S_{a b}$ is an average value of the Reynolds stress between along beam positions $a$ and $b$. 
Evaluation of the Reynolds stress using Eq. (8) requires specification of the wave attenuation parameter, $\beta$. We assume this to be time invariant and defined as:

$$
\beta \approx \sqrt{\frac{\overline{\tilde{u}_{b}^{2}}}{\overline{\tilde{u}_{a}^{2}}}}
$$

where $\overline{\tilde{u}_{a}^{2}}$ and $\overline{\tilde{u}_{b}^{2}}$ are the variances at $a$ and $b$ due to the wave motion. The wave variances are computed from linear wave theory using wave parameters determined by fitting a model along beam variance profile, $\overline{\tilde{u}_{\text {beam }}{ }^{2}}$, to the observed along beam variance profile. The model variance profile is obtained by substituting linear theory expressions for $\tilde{u}$ and $\tilde{w}$ (Dean and Dalrymple, 2002) into Eq. (2), evaluating the time averages and simplifying:

$$
\overline{\tilde{u}_{\text {beam }}^{2}}=\left(-\frac{H^{2} \omega^{2}}{16}\right)[\operatorname{csch}(k h)]^{2}[\cos (2 \theta)-\cosh 2 k(h+z)]
$$

where $H$ is wave height, $h$ is mean water depth, and $z$ is the vertical position which is positive up and zero at the surface. The wave parameters, $H, \omega$ and $k$ were obtained by fitting Eq. (10) to the observed velocity variance along the axis of each ADCP beam. A single value of wave parameters was determined for each beam pair using an average of the values determined from the fits to the corresponding beams. Given these wave parameters, $\overline{\tilde{u}_{a}^{2}}$ and $\overline{\tilde{u}_{b}^{2}}$ were obtained using Eq. (10) at vertical positions $z_{a}$ and $z_{b}$, respectively.

The validity of this procedure relies on the majority of the observed variance being due to wave motion. Our deployment data bear this out extremely well. Figure 3 shows several examples of model fits covering a range of wind, wave, and velocity variance activity. Typically the largest and most frequent deviations from the model 
variance profile occurred

near the surface. Therefore,

the near surface data were

excluded in the profile fits

by eliminating points one at

a time, beginning at the

surface, until a local

maximum in $r^{2}$ was

achieved. If no local

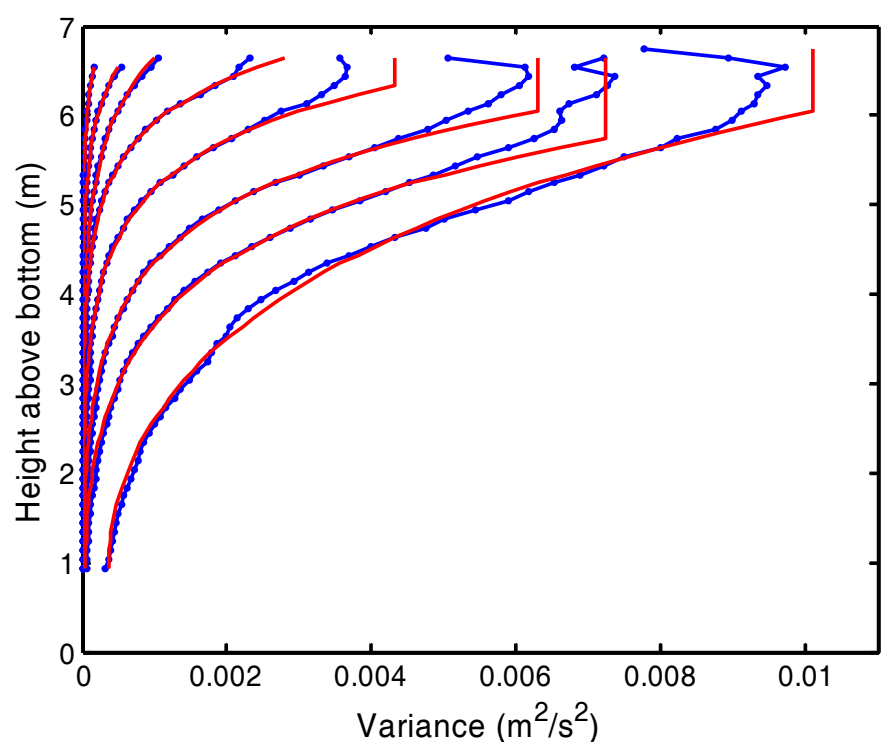

Figure 3. Several typical observed velocity variance curves are maximum was obtained, the

search was abandoned after

shown in blue with the model wave curve fits overlaid in red. The curve fits ignore near surface data that is obviously not consistent with linear wave theory.

removing the top $2.5 \mathrm{~m}$ of the water column. (This distance was based on a visual inspection of velocity variance profiles in the water column.) This occurred extremely infrequently (5 times out of over 3000). Thus, in addition to providing an estimate of the wave parameters and $\beta$, this procedure allowed us to compute the depth of the near surface zone within which linear wave theory did not account for the dominant velocity variance. The times when the near surface velocity variance departed most strongly from a pure wave profile were periods of greatest winds and waves, steepest variance profiles, and greatest mixing and momentum exchange. We assume this behavior reflects the presence of enhanced turbulence from breaking waves and that the depth of the surface zone within which linear theory did not account for the dominant velocity variance is related to the penetration depth of the turbulence effects of breaking waves.

The best fit values of $H, \omega$ and $k$ were used to determine $\beta$ over the majority of the water column using Eqs. (9) and (10). However, due to the obvious departure of the near 


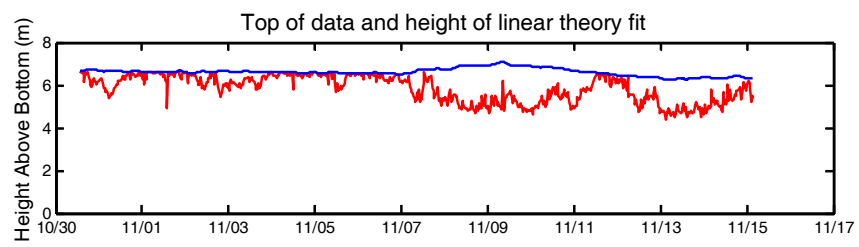

surface variance field from

that of linear waves, we

abandon this procedure near

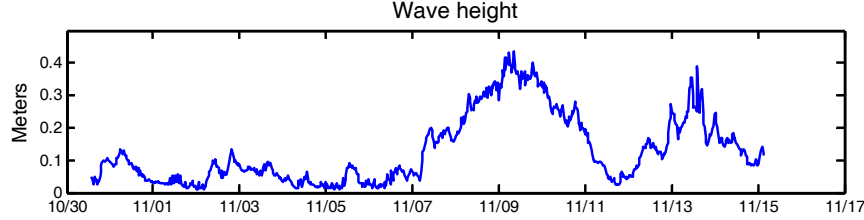

surface and rather held the

value of $\overline{\tilde{u}_{\text {beam }}^{2}}$ constant in this

region (see Figure 3).

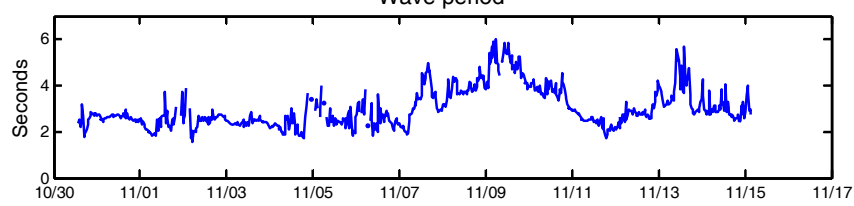

Figure 4 summarizes

the computed wave

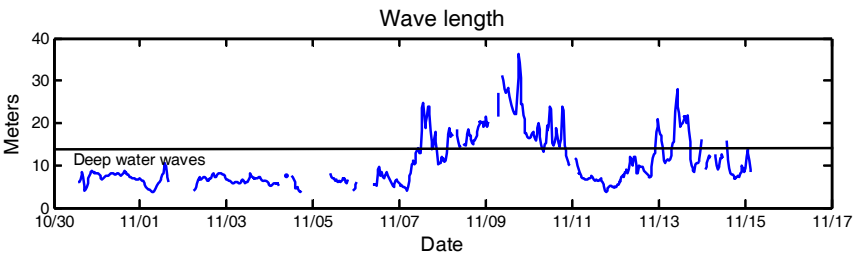

parameters, the range of the near surface zone and the total water elevation (less 6 percent for ADCP side-lobe

Figure 4. The top panel shows the height of the near surface zone (red) where the linear wave theory based model did not realistically account for the wave associated variance as compared to the total water elevation (less 6\% for ADCP side lobe contamination, blue). The second through fourth panels show mean wave height, mean wave period and wavelength (in the direction of wave propagation) as determined by the curve fitting procedure described in the text. The black line on the bottom panel divides wavelength into regions of deep-water waves where the wavelength is beneath the line and intermediate waves above. Shallow water waves would occur at a wavelength of around 140 meters.

contamination) during the

course of the 15 day

deployment. The black line

in the fourth panel indicates the transition from deep-water waves (wavelengths below the line) to intermediate waves (above the line). This threshold occurs when the wavelength is less than twice the depth (Brown et al., 1989). Shallow water waves, given as wavelengths greater than twenty times the depth, would start with wavelengths around 140 meters and do not exist in this dataset. Despite the rather shallow $(7 \mathrm{~m})$ water depth, the short wave lengths resulted in waves that felt minimal influence from the bottom at the deployment location. 


\section{Results}

Application of this wave removal method requires selection of a separation distance for the differencing (i.e., the distance between positions $a$ and $b$ ). This distance should be large enough that turbulent components of the velocities are uncorrelated between $a$ and $b$ (Eq. (6)), yet small enough that the Reynolds stress does not vary significantly between $a$ and $b$ (Eq. (7)). After some experimentation, we selected a vertical separation distance of $1 \mathrm{~m}$ as a compromise between these somewhat conflicting requirements. An assessment of this selection is presented below.

The effectiveness of the wave-turbulence separation is first assessed by examining along beam velocity spectra and Reynolds stress spectra before and after the separation is applied. Figure 5 presents these spectra for a pair of beams at two elevations (near surface and mid-depth) and four different times during the deployment. In general, the peak in each raw spectrum lies in the range of $0.3-0.5 \mathrm{~Hz}$ and is presumably associated with surface gravity waves.

During three of the four periods, (Nov 7, 10,11) winds were light to moderate with sustained magnitudes of 5-7 m/s (see Figure 9). Waves are apparent in the near surface and mid-depth spectra peaking near $0.5 \mathrm{~Hz}$. The wave-turbulence separation procedure cleanly eliminates this portion of the along beam velocity spectra and the associated apparent Reynolds stress with little effect on the lower frequencies. We note that these periods represent intervals of modest wave formation as well as periods of wave decline.

During the period of strongest winds (about $12 \mathrm{~m} / \mathrm{s}$, Nov 9) the wave peak appears to occur at lower frequency and the energy of the lower part of the velocity spectra (e.g., frequencies less than $0.2 \mathrm{~Hz}$ ) is nearly an order of magnitude greater than during the other times. In this case the wave separation procedure eliminates what appears to be the wave 

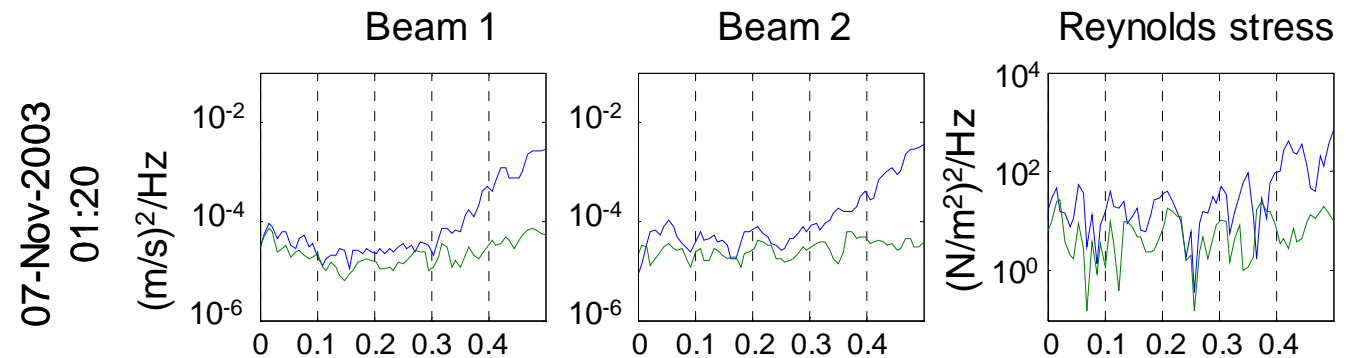

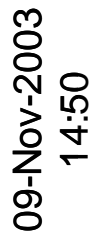
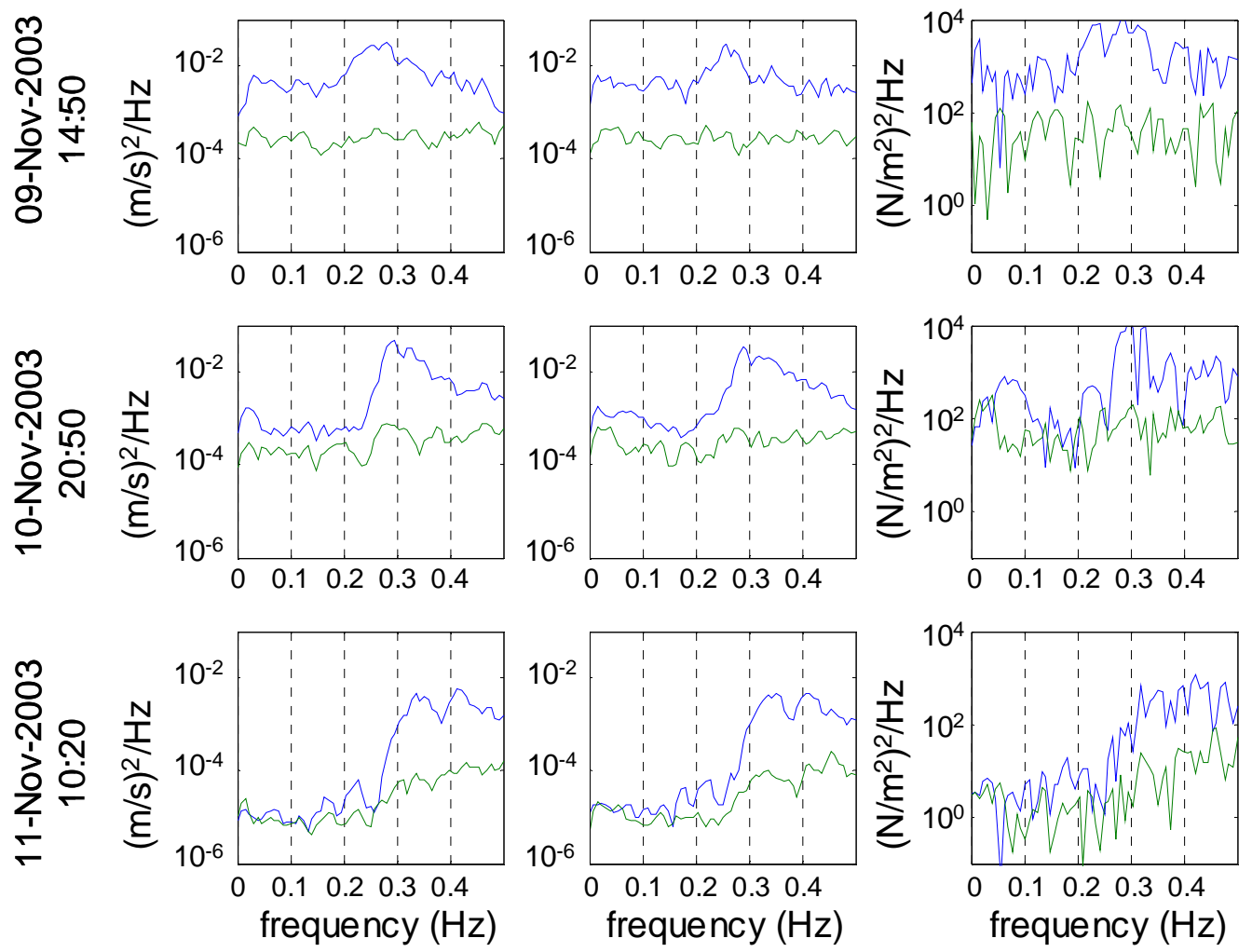

Figure 5a. Spectra of along beam velocity and Reynolds stress for raw data (blue) and waveturbulence separated data (green) for one pair of ADCP beams at four different times (rows). Results at 5.24 meters above bottom. 

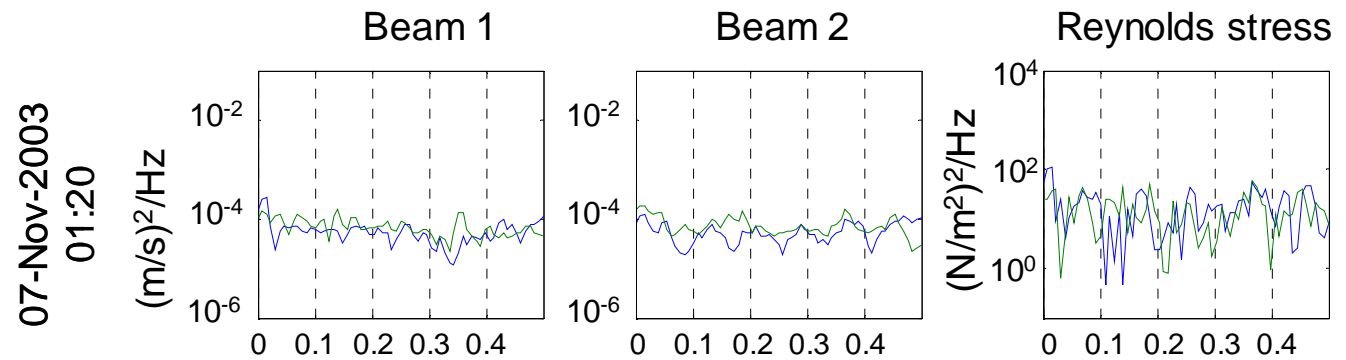

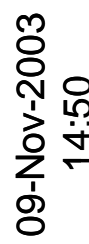
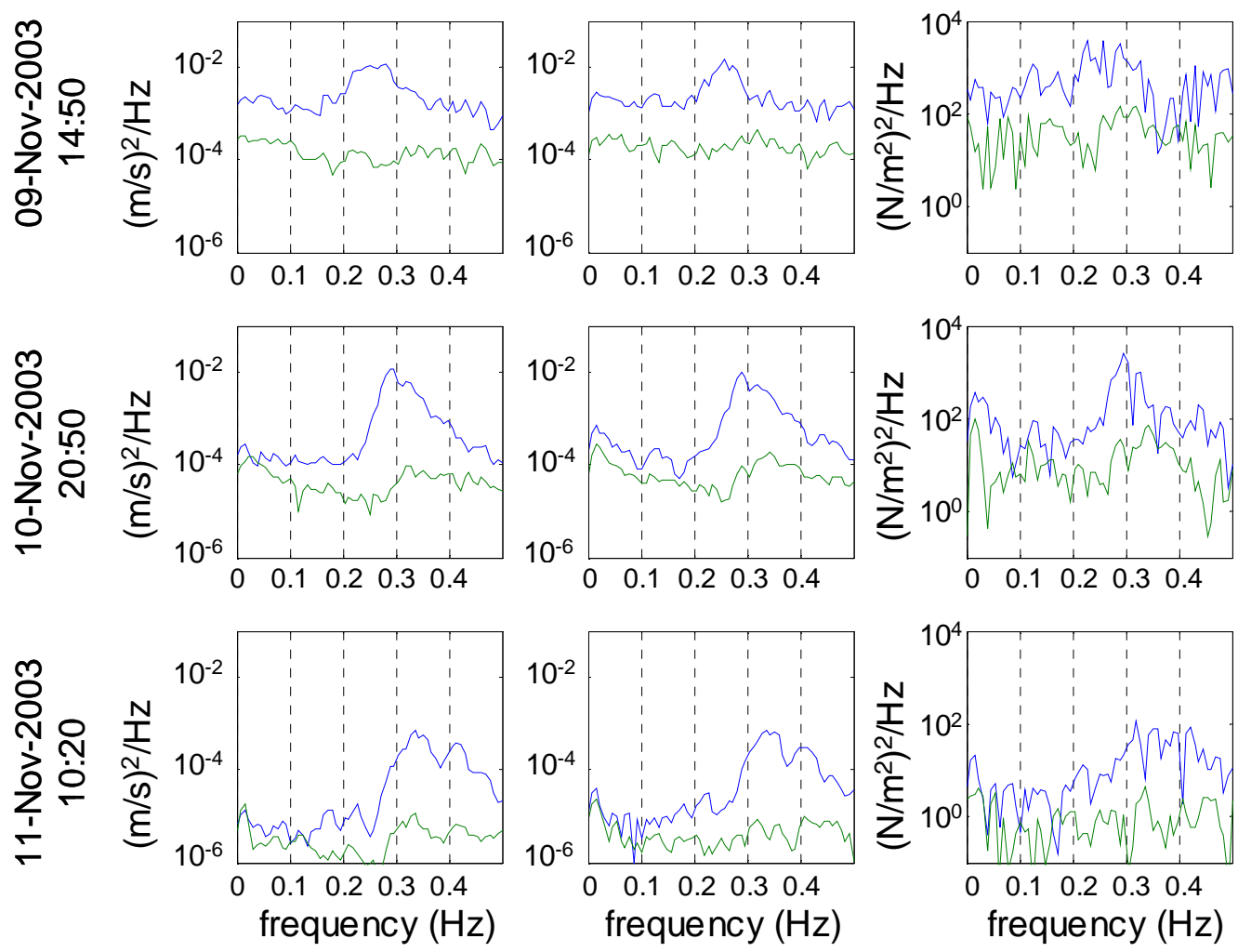

Figure 5b. Spectra of along beam velocity and Reynolds stress for raw data (blue) and waveturbulence separated data (green) for one pair of ADCP beams at four different times (rows). Results at 3.34 meters above bottom. 
band component of the spectra, but it also lowers the spectra across all frequencies. At this point it is not clear whether this reflects more broadly banded wave motion or undesired filtering of the turbulence, perhaps due to partial coherence between the signal at levels $a$ and $b$.

The influence of the scaling factor for the vertical subtraction, $\beta$, on these results is demonstrated in Figure 6, by computing the along beam velocity spectra as $\beta$ is varied over its maximum possible range (from 0 to 1 ). The top and bottom panels correspond to the second and fourth rows of Figure 5a; the $\beta$ values determined by fitting the linear theory model to the variance profiles (and used in the corresponding panels in Figure 5a) are 0.8 and 0.5 . In both cases these values of $\beta$ correspond very well to minima in the energy in the wave portion of these spectra. Thus it appears that the selected $\beta$ values are optimal for removing

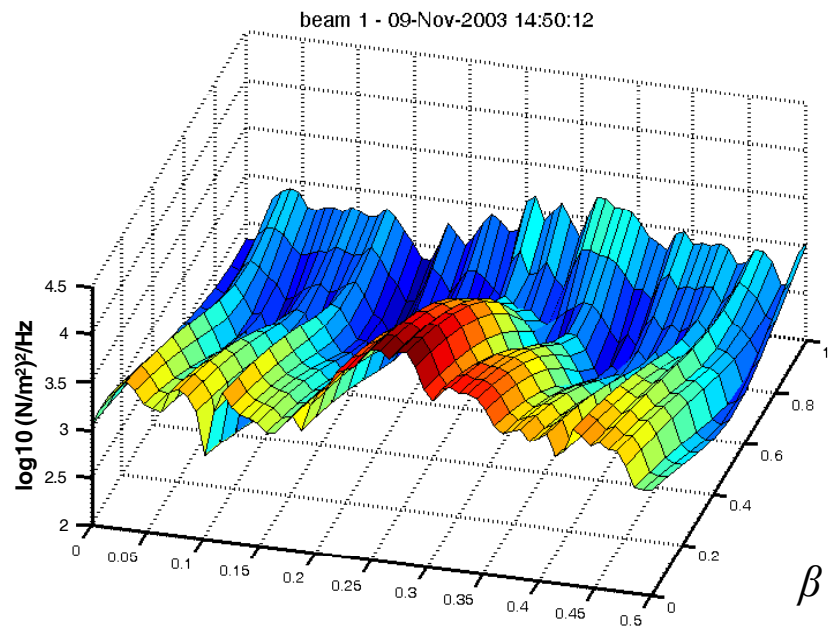

frequency $(\mathrm{Hz})$

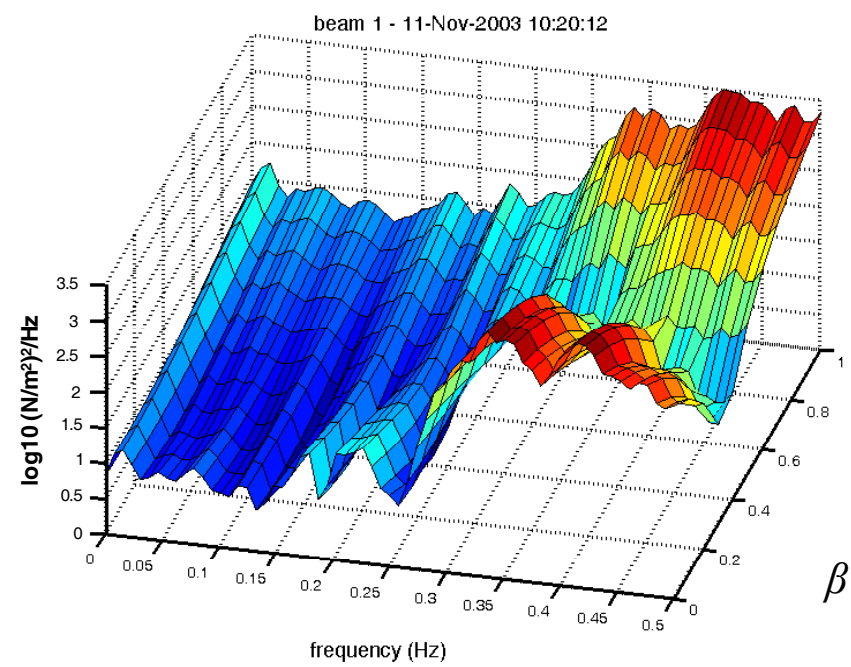

Figure 6. Near surface (5.24 mab) along beam velocity spectra for values of $\beta$ ranging from 0 to 1 at two of the times shown in Figure 5. $\beta$ values obtained by fitting the variance to a model based on linear wave theory are 0.8 and 0.5 in the upper and lower panels, respectively. wave energy. 

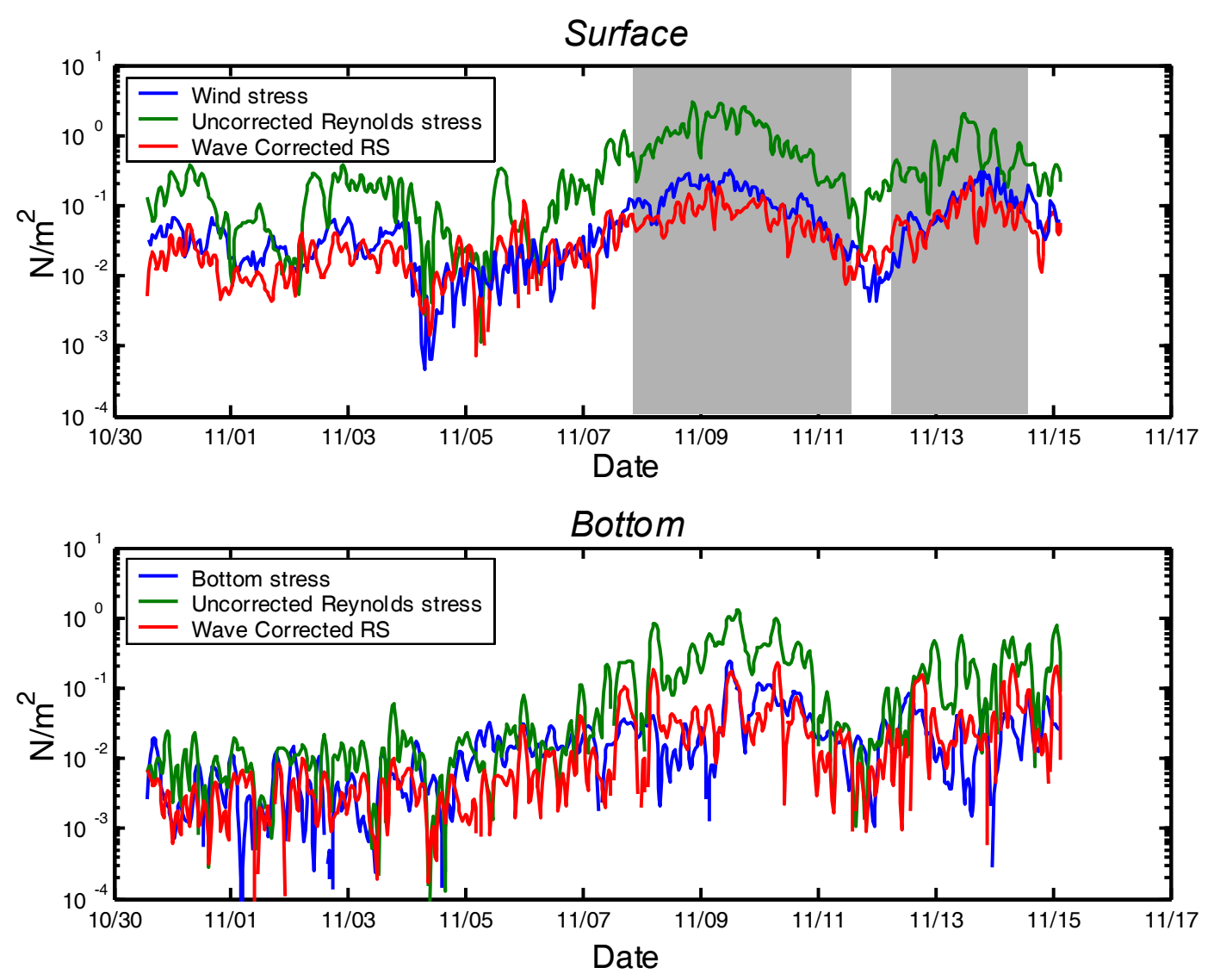

Figure 7. The top panel shows surface wind stress computed using a drag law compared to the magnitude of the uncorrected Reynolds stress and to the magnitude of the Reynolds stress after the wave correction was applied. Shaded areas correspond to times when the surface Reynolds stress values were computed in a region of the water column where the linear wave theory based model provided a poor fit to the velocity variance profile. The bottom panel makes the comparison of the bottom stress computed using a drag law to the near bottom Reynolds stress computed with and without the wave correction.

Figure 7 compares the magnitude of the surface and bottom stress values as predicted by drag laws to the magnitude of both the uncorrected and the wave separated Reynolds stress. Note that the wave-separated Reynolds stress values are for about a meter below the surface. This is by nature of the method where $6 \%$ of the water depth was removed due to ADCP side lobe contamination, and the $1 \mathrm{~m}$ vertical subtraction distance lowered the mean position of the Reynolds stress calculation another half meter. Uncorrected Reynolds stresses are plotted at the same level for comparison. The bottom 
stress was computed at the lowest possible depth in the water column, which is a halfmeter above the lowest bin or roughly $1.4 \mathrm{~m}$ above bottom. Computed Reynolds stress values were filtered in time to reduce noise using a $15^{\text {th }}$ order Butterworth filter with a normalized cutoff of 0.3 (yielding roughly a 3-point, or hour and a half average). The wind stress was computed for comparison using an air-sea Matlab toolbox following Large and Pond (1981). Bottom stress was computed for comparison using a drag coefficient of $3 \times 10^{-3}$ times the square of the velocity of the lowest bin, which was 0.94 meters above bottom. A constant bottom drag coefficient is reasonable since the waves were nearly deep-water waves (e.g. Figure 4) and consequently had minimal impact on bottom drag.

The wave-separated Reynolds stresses agree quite well with values predicted by drag laws, Figure 7. Examining the bottom stress record, it can be seen that where there is little wave action, there is minimal difference between the drag law prediction, the uncorrected and the wave-separated Reynolds stresses. During the stronger wind events that occur around Nov. 9 and 13, the un-corrected Reynolds stresses increase in response to contamination by wave motions reaching the bottom, while the wave-separated data still track the drag law prediction closely. At the surface the distinction between the corrected and uncorrected Reynolds stresses is even clearer. The corrected data follow the wind stress fairly closely while the uncorrected data are typically an order of magnitude too large. The shaded areas correspond to times when the near surface Reynolds stresses are computed in the part of the water column where the velocity variance profiles were poorly modeled using linear wave theory (i.e. above the red line in Figure 4). Values in this region track the change in wind stress reasonably well but tend to dip under the drag based computations. The most likely explanation for this may be the $1 \mathrm{~m}$ separation distance being less than the turbulence correlation length scale, thereby causing some of 
the Reynolds stress to be filtered out by the wave correction. This is also consistent with the broad banded reduction in the turbulence spectra during this period (Figure 5).

If the wave separation process is selectively removing wave-induced apparent Reynolds stresses, it is expected that the difference between the uncorrected and the corrected Reynolds stresses would be correlated with the wave height. A cross covariance was run comparing this difference with wave height. The normalized cross covariance value at zero lag was 0.76 indicating that the wave separation had the greatest effect during periods of greatest wave activity.

The error in Reynolds stress due to wave motion caused by instrument tilt was assessed following Rippeth et al. 2003. Using linear wave theory an error term for the Reynolds stress was calculated based on data from the instrument tilt sensors and wave parameters as shown in Figure 4. Figure 8 shows this error term compared to the
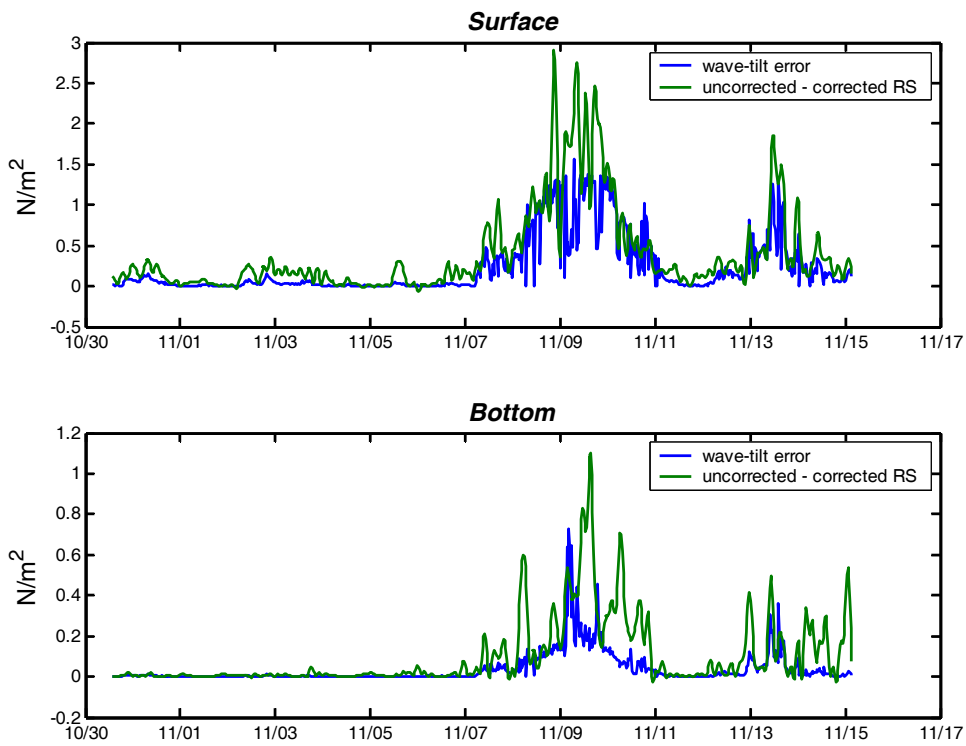

Figure 8. The error in un-corrected Reynolds stress due to the interaction of wave motion with instrument tilt is shown in blue. The difference between the uncorrected and corrected (waveseparated) Reynolds stress is shown in green. The top panel is near surface and the bottom panel is near bottom. difference between the corrected and un-corrected Reynolds stress for both the near surface and near bottom locations shown in Figure 7. It is clear that the wave separation process removed an amount of apparent Reynolds stress that corresponds closely

with the error predicted to occur due to the interaction of waves and tilt. 
Figure 9 shows profiles of density and water velocity at the study site, and wind vectors collected from a C-MAN station approximately $45 \mathrm{~km}$ to the south. Data have been rotated into downstream and across channel components using a principal
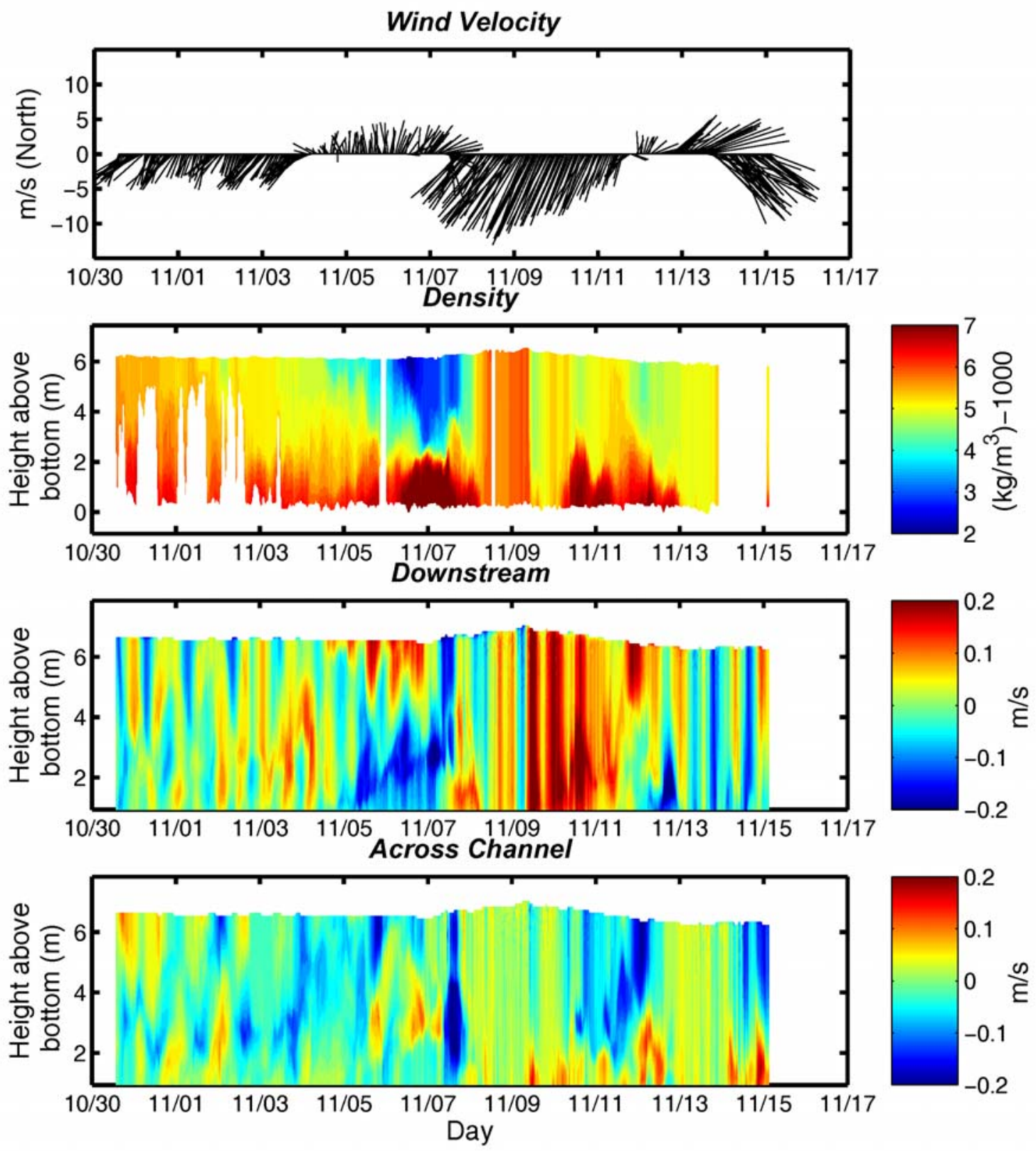

Figure 9. The top plot shows wind vectors (direction wind is blowing toward, north up) from a C-MAN station approximately $45 \mathrm{~km}$ south of the data collection site. The second plot gives profiles of density during the data collection period (white areas are missing data). The third plot shows water velocities rotated into the along stream direction with positive downstream. The fourth plot shows water velocities in the across channel direction with positive toward the northwest. 
components analysis of the depth-averaged velocities for the entire dataset. The density record shows that stratified conditions exist frequently and are enhanced by downstream blowing winds. Full vertical mixing occurs when winds exceed approximately $10 \mathrm{~m} \mathrm{~s}^{-1}$, e.g., on Nov. 8-9 and again on Nov. 13. Classical estuarine flow can be observed in the velocity record during much of this period and is especially notable during Nov. 5-7 with surface water moving downstream and denser bottom water moving upstream. Regular oscillations are frequently seen in the velocity record and represent the 13.2-hour barotropic seiche that is pervasive in the Pamlico Sound system (Luettich et al, 2001). From the latter half of Nov. 9 through the beginning of Nov. 11, most of the water column was moving downstream (positive velocities) while the wind stress was directed upstream. During this period the forcing due to the elevation setup, (both wind and river flow driven) along the axis of the sound and into the Neuse River Estuary was apparently stronger than that due to the wind stress and therefore able to drive this downstream flow throughout most of the water column. We expect the flow very close to the surface was more closely aligned with the wind direction, although this apparently occurred within the surface blanking zone of the ADCP (roughly the upper half meter of the water column).

The Reynolds stress components in the downstream and across channel directions were computed throughout the water column using the wave correction procedure and are shown in Figure 10. Overlaid on the plot is the line representing the elevation in the water column to which the linear theory based wave variance model fit the observational data, as also shown in Figure 4. It is striking how well this line delineates a change in the character of the Reynolds stresses, presumably due to the effects of breaking waves on the turbulence field. Below the line the Reynolds stresses track the wind quite well. For example upstream blowing winds on Nov. 8-11 and downstream blowing winds on Nov. 13-15 are well represented by similarly directed Reynolds stresses. There is evidence of 

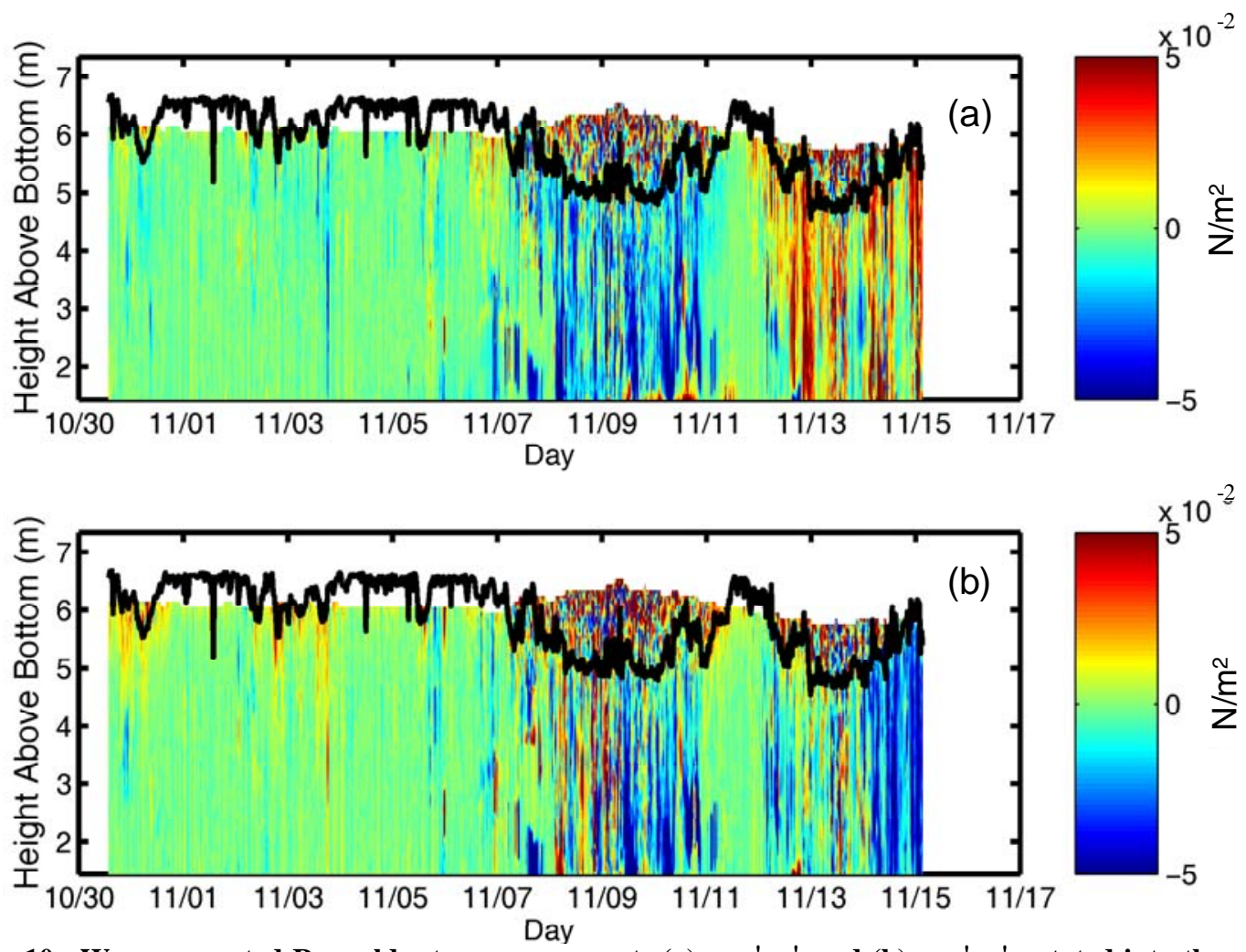

Figure 10. Wave corrected Reynolds stress components (a) $-u$ ' $w^{\prime}$ and (b) $-v^{\prime} w^{\prime}$ rotated into the along channel and across channel directions respectively. Overlaid is the upper extent of the wave fit curves.

positive Reynolds stress near the bottom (recall Figure 10 is limited to approximately 1.5 m and higher above the bottom) during Nov. 9-11, which is presumably associated with bottom stress due to the downstream barotropic flow throughout the water column. The Nov. 7-11 and Nov. 13 winds have relatively small across stream components and the corresponding across stream water column stresses are low and variable. However, on Nov. 14 the winds develop a strong across stream component and this is reflected in a strong across stream Reynolds stress.

The close directional consistency between the Reynolds stresses, the wind forcing and the barotropic flow does not hold in that portion of the water column above where the linear theory based model fits the observed variance data during the Nov. 8-11 wind event. During this period the computed Reynolds stresses are quite noisy in direction with a somewhat positive bias (as opposed to the negative bias that would be expected 
from an upstream directed shear forcing at the surface). Despite trying numerous variants from our wave - turbulence separation procedure, we have been unsuccessful devising a method that corrects the directional inconsistency in this region or that fully explains its origin. As discussed previously, it is interesting that the stress magnitude during this period remains consistent with, albeit slightly below, the computed wind stress.

Figure 11 shows the gradient Richardson number and the magnitude of the Reynolds stress. For display clarity, buoyancy frequencies, and consequently Richardson
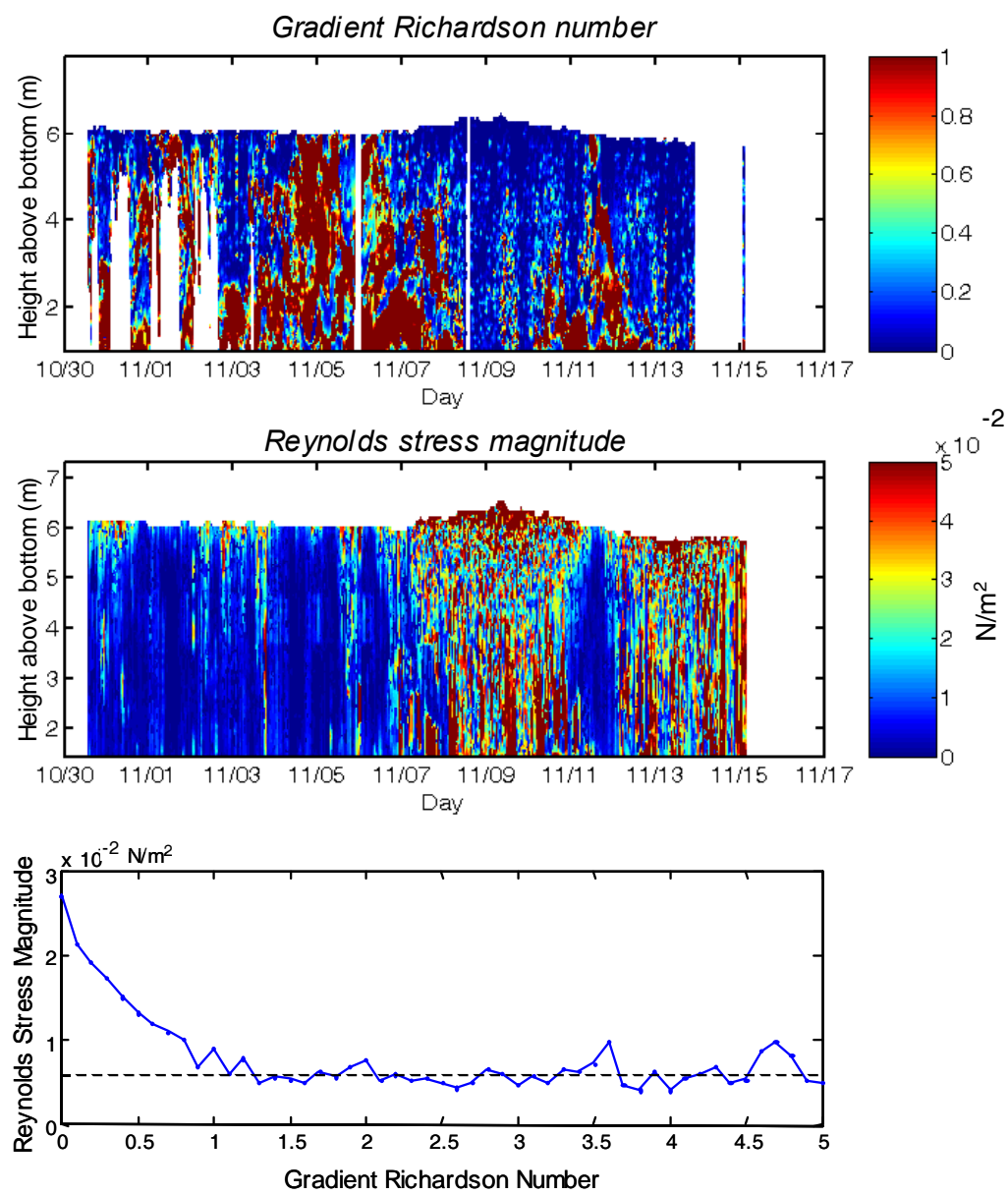

Figure 11 The top panel shows the gradient Richardson number. The second panel shows the magnitude of the Reynolds stress (the vector sum of the $\overline{u^{\prime} w^{\prime}}$ and $\overline{v^{\prime} w^{\prime}}$ components). The third panel shows the gradient Richardson number verses Reynolds stress magnitude. The horizontal dashed line represents the lower Reynolds stress measurement threshold based on instrument noise (Williams and Simpson, 2004). 
numbers, were not allowed to become negative. Extremely small values of shear also made the calculation unstable. Consequently values of the shear-squared below $5 \mathrm{e}-4 \mathrm{~s}^{-2}$ were taken as the average of their vertically adjacent neighbors. Comparing the two panels shows that, as expected, the turbulence coincides well with periods of low Richardson number. To further demonstrate this, data were binned by values of Richardson number in increments of 0.1 and the mean of the Reynolds stress was calculated per bin (Figure 11). Reynolds stress is clearly a monotonically decreasing function of Richardson number for Richardson numbers between zero and about one. The noise floor for measuring Reynolds stress was calculated using Eq. 14 from Williams and Simpson (2004) and found to be $0.6 \times 10^{-2} \mathrm{~N} / \mathrm{m}^{2}$. Thus Reynolds stress values for Gradient Richardson numbers greater than about one are at the level of the instrument noise. This is consistent with shear generated turbulence theory although the cutoff Richardson number is a bit larger than the theoretical value of 0.25 . Factors that would contribute to the loss of precision in the Richardson number include the discrete nature of both the buoyancy frequency measurement and the velocity shear measurement, the physical separation of these measurements of about 10 meters, the different sampling interval of the ADCP and the vertical density profiles, and the inability to perfectly match depth bins from the profiler to height above bottom bins from the ADCP.

An analysis of the uncertainty in the Reynolds stress was done following Williams and Simpson (2004) assuming $\gamma_{R}=1$. Figure 12 shows the Reynolds stress plotted versus the standard deviation of the Reynolds stress. In the top panel color identifies the number of good observations (out of a possible 900) returned by the ADCP during each 15minute sampling period. As the number of good observations approaches 900 for each sampling period, the uncertainty, as indicated by the standard deviation of the Reynolds stresses decreases significantly as compared to sampling periods with fewer good 

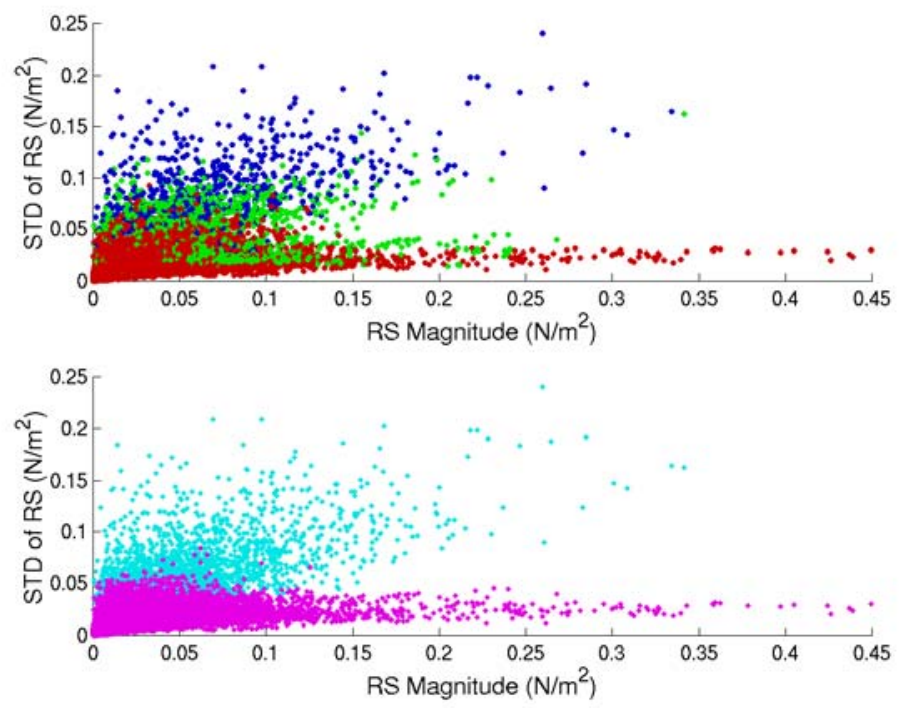

Figure 12 Reynolds stress plotted as a function of the standard deviation of the Reynolds stress. In the top panel color identifies the number of good observations returned by the ADCP. Out of a maximum of 900 , red indicates $700-900$, green indicates 500 699, and blue indicates less than 500 good observations. In the bottom panel cyan indicates data in the portion of the water column where the variance profiles were poorly modeled using linear wave theory (i.e. above the red line in Figure 4). Magenta indicates data that were well fit by linear wave theory (i.e. below the red line in Figure 4). observations. The bottom

panel shows data separated into values where the variance profiles were poorly modeled using linear wave theory (i.e. above the red line in Figure 4), and values that were well fit by linear wave theory (i.e. below the red line in Figure 4). It is quite clear that the uncertainty in the Reynolds stress is much higher in the upper region.

\section{Potential Limitations of ADCP Mode 11}

The ADCP data presented in this study was collected using the relatively new mode 11 sampling scheme. The benefit of using mode 11 is that it has 10 to 100 times the single ping precision of standard modes. However, this comes with limitations on its profiling range, maximum velocity and maximum velocity shear. We experienced high data return from the entire water column during much of our deployment. The data return dropped off significantly near the surface during periods of strong wind and waves and to a lesser extent throughout the water column during higher energy periods, Figure 13. A consequence of this data dropout is that we may have systematically missed the more energetic or high shear parts of the velocity signal and thereby biased our results to under represent derived quantities such as the actual Reynolds stress in these higher-energy 

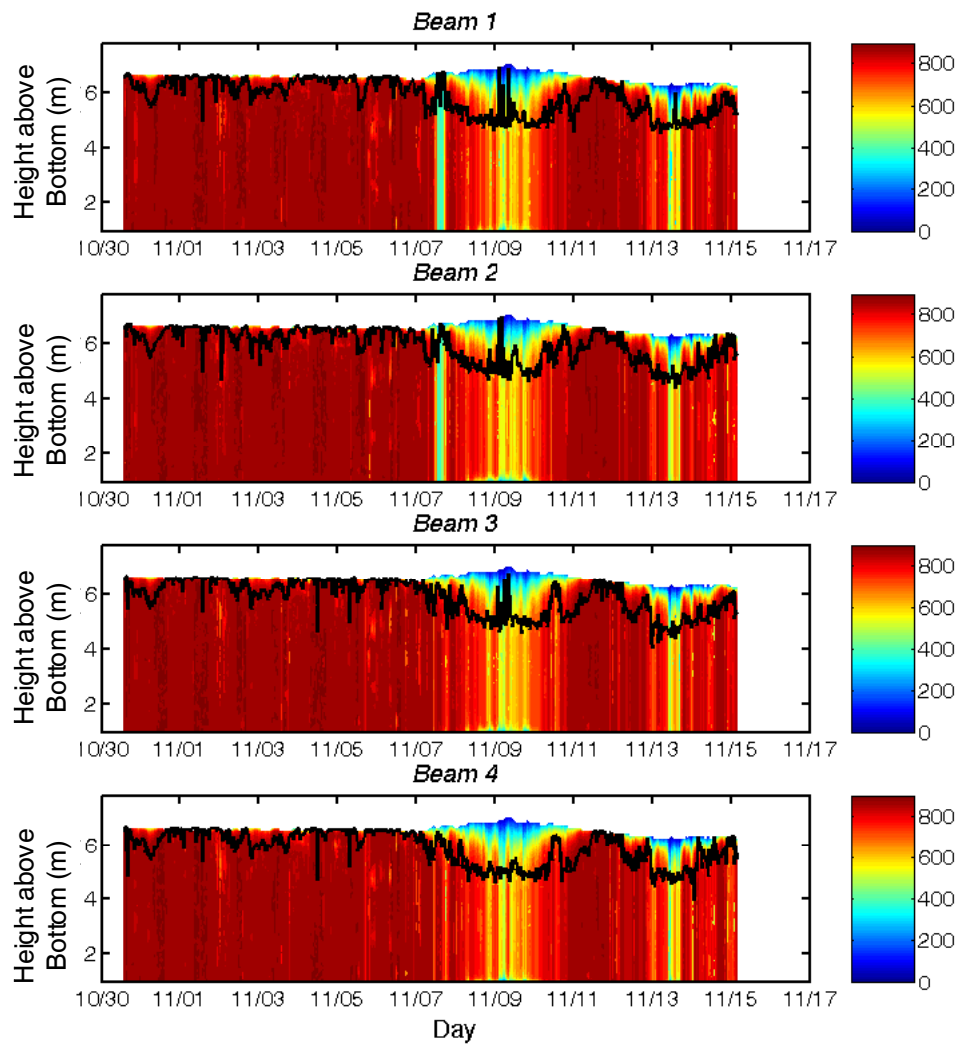

Figure 13 The number of good data values during each 15-minute sampling period (out of a possible 900). Data are shown for each of the four beams. The black line is the height of the wave fit curves for each period.

periods. If this was the case, we might expect to see evidence of truncation of the velocity signal or shear at the high end, indicating instrument response saturation. However, plots of the distributions of along beam velocity and along beam shear, during periods of significant data drop out, do not provide any indication of such response saturation, Figure 14. In addition, the variance profiles (e.g., Figure 3) indicate the existence of a significant dynamic range in the variance in the high energy near surface region. Had the velocities saturated in this region, we would have expected many of these variance profiles to converge to a common upper limit. We saw no indication of this during the deployment (nor two other deployments that have not been included in this presentation). Thus, there is reasonable indication that instrument saturation was not a systematic problem in these data.

We note that there is not a tight correspondence between the ADCP data return and the elevation of the linear wave theory based model fit, (Figure. 13). Therefore data 

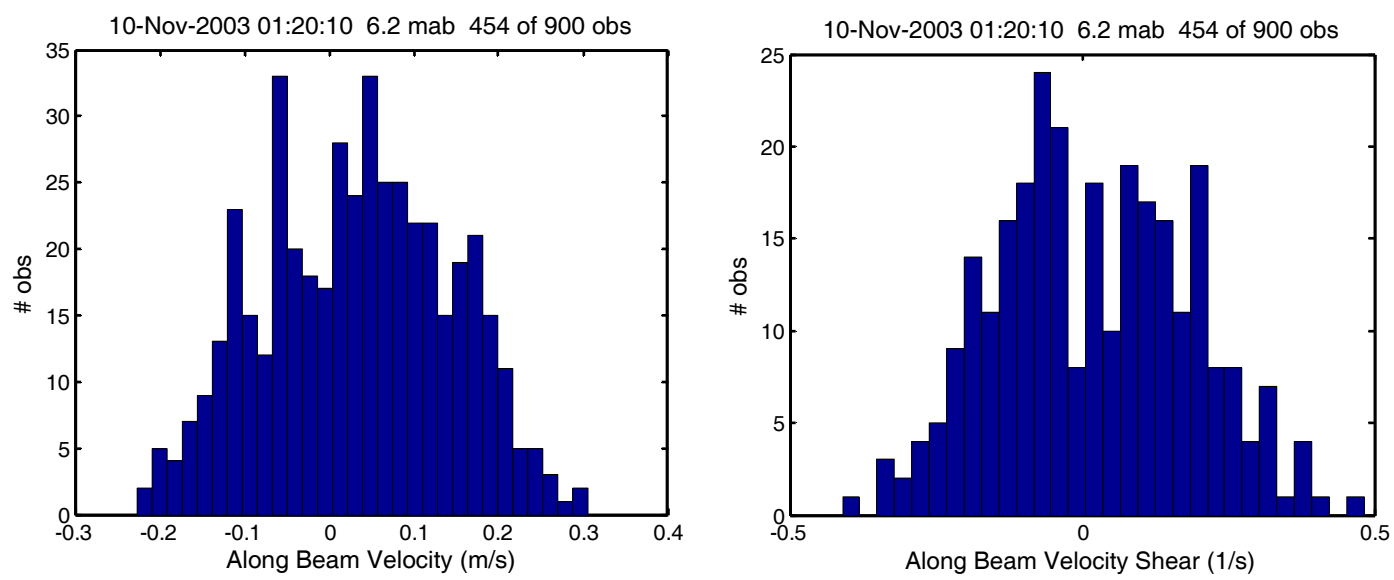

Figure 14 Typical distributions of velocity (a) and shear (b) near the surface during a period of high winds and high data loss from the ADCP.

dropout does not appear to be a definitive explanation of the noisy Reynolds stress directions we observed above this elevation.

\section{Effect of the Vertical Separation Distance in the Wave - Turbulence Separation}

The wave removal method requires selection of a separation distance for the differencing that is large enough that turbulent components of the velocities are uncorrelated between the two locations (allowing elimination of the final two terms in Eq. (5)) yet small enough that the wave velocities are perfectly correlated and that computing an average value of the Reynolds stress is appropriate over this distance. A somewhat subjective decision was made to use a vertical separation of $1 \mathrm{~m}$ as a compromise between these potentially conflicting constraints.

We note that independent knowledge of the final two terms in Eq. (5) would eliminate the uncertainty that is imparted to this method by the separation distance. While our expression for Reynolds stress, Eq. (8), is based on the limit of no correlation in the turbulent velocity over the separation distance, an expression can also be developed for the limit of perfect correlation in the turbulent velocity over this distance. In this case the Reynolds stress is: 


$$
R S_{a b} \approx \frac{\overline{\Delta u_{3}^{2}}-\overline{\Delta u_{4}^{2}}}{4 \sin \theta \cos \theta(\beta-1)^{2}}
$$

Eq. (11) offers a possible alternative method for computing Reynolds stress by differencing closely spaced data in which the perfect correlation assumption is reasonable. In addition, the ratio of Eq. (11) to Eq. (8):

$$
\frac{\text { correlated } R S_{a b}}{\text { uncorrelated } R S_{a b}}=\frac{\beta^{2}+1}{(\beta-1)^{2}}
$$

provides an idea of the sensitivity of the computed Reynolds stress to the assumption that the turbulence is not correlated over the separation distance. In this worst case scenario, the Reynolds stress would be incorrect by as much as a factor of 10 over the typical values of $\beta$ encountered in this study, Figure 15.

Vertical turbulent fluctuations with scales appreciably greater than the separation distance should be attenuated or removed by the wave - turbulence separation process
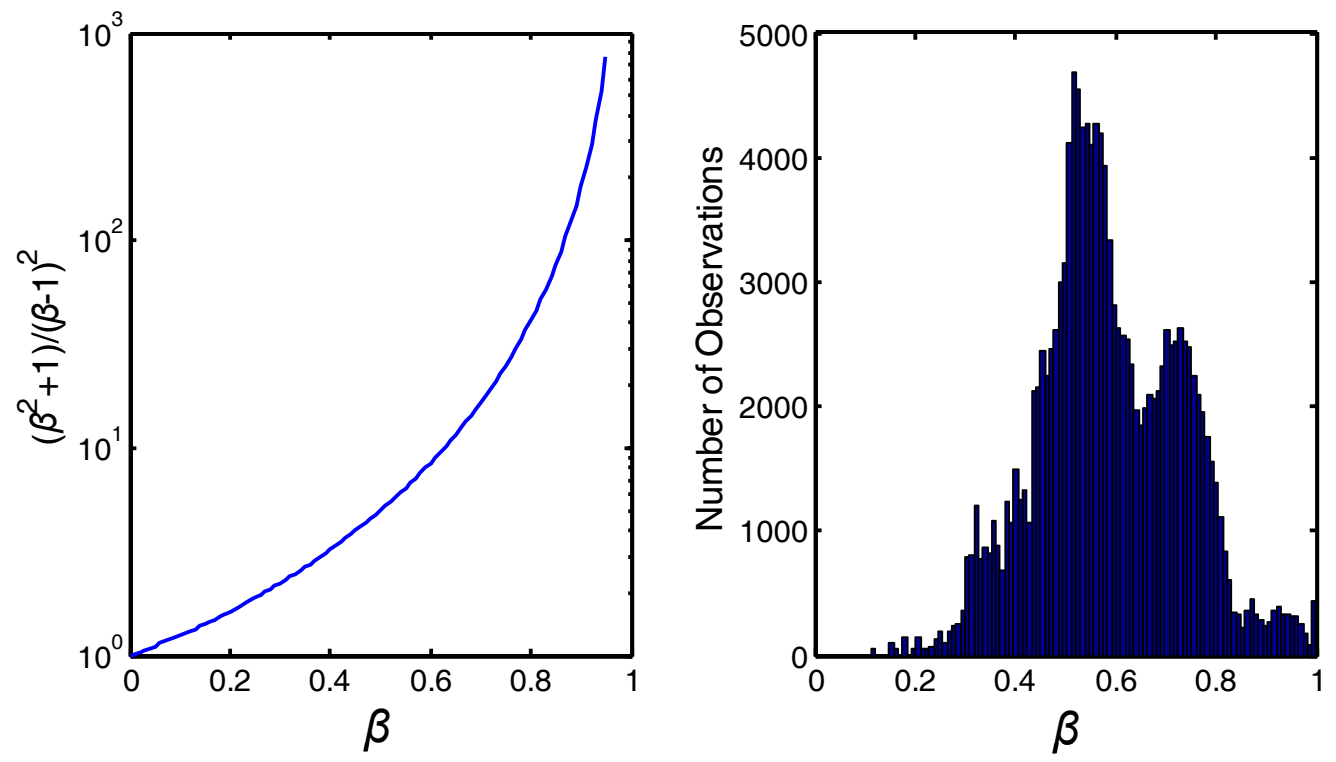

Figure 15 Ratio of Reynolds stress assuming perfectly correlated turbulence over the separation distance vs uncorrelated turbulence over this distance, (ratio of Eq. (11) to Eq. (8)), and the distribution of $\boldsymbol{\beta}$ values for this dataset. 
and the resulting Reynolds stresses

would be under-estimates of the true

values. Figure 16 provides an

indication of the impact of the

separation distance varying between

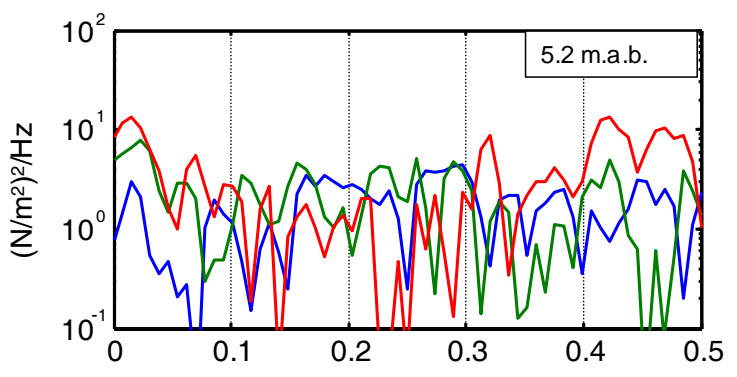

$0.5 \mathrm{~m}-1.5 \mathrm{~m}$ on the Reynolds stress

averaged over the entire sampling

period. The $0.5 \mathrm{~m}$ spacing clearly

reduces the magnitude of the Reynolds

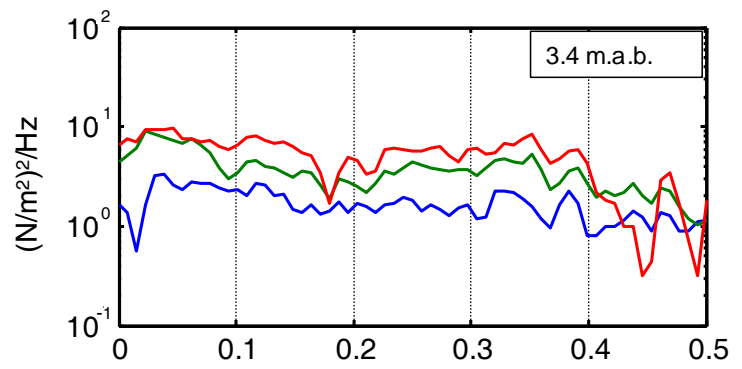

stresses while there is little difference

between the $1 \mathrm{~m}$ and $1.5 \mathrm{~m}$ spacing,

supporting our use of a $1 \mathrm{~m}$ separation.

The optimal separation should clearly

be a function of the characteristics of

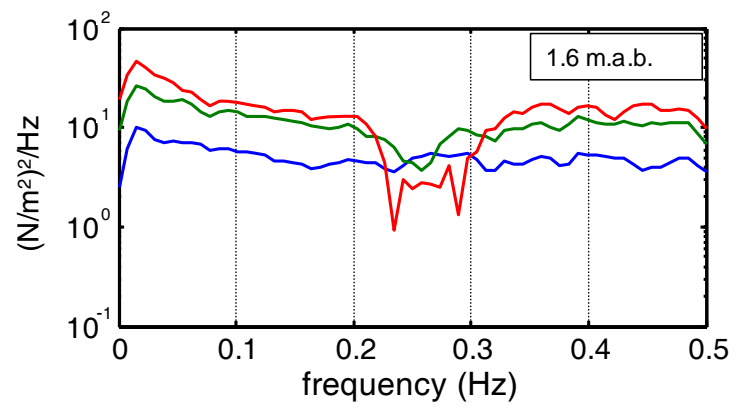

the turbulent flow field and therefore

this distance would need to be re-

Figure 16 Reynolds stress spectra averaged over the entire study period for three separation distances, 0.5 $\mathrm{m}$ - blue, $1.0 \mathrm{~m}$ - green, $1.5 \mathrm{~m}$ - red, at three different assessed for other applications of this heights in the water column.

wave - turbulence separation methodology. To estimate the magnitude of the turbulent length scales in our data we can refer to Stacey et al. (1999) who employed multiple techniques to establish length scales in their data. In 9 meters of unstratified water they found length scales at mid-water to be on the order of 1-2 meters depending on the method. Since our data are from 7 meters of water that is often stratified, we would expect our length scales to be in the same size range or smaller. 


\section{Conclusions}

The variance technique has been used successfully in tidally dominated flows to calculate Reynolds stresses from a 4-beam ADCP. However, surface wave contamination is a significant source of error in this and other methods for determining Reynolds stresses. We have proposed a technique for separating wave and turbulent motions for use with the variance method by subtracting along beam ADCP velocities over a specified separation distance after scaling to account for the decrease in wave orbital velocity with depth.

We applied this approach in a shallow (7 m), wind driven, lagoonal estuary using a $1200 \mathrm{kHz}$ ADCP and the “mode 11” sampling algorithm for a 15.5 day deployment period. While there was increased ADCP signal rejection near the water surface during periods of strong wind and wave activity, there was no apparent saturation of the velocity data as a result of sampling with model 11. In most cases, the wave - turbulence separation selectively reduced the power in the spectral band occupied by the surface waves. Reynolds stresses computed during periods of significant waves were reduced by approximately an order of magnitude over values computed without making the wave turbulence separation. The magnitudes of the corrected Reynolds stresses agreed quite closely with values predicted using drag laws at the surface and bottom throughout the deployment. A comparison between the Reynolds stresses removed by the wave turbulence separation and the error in the Reynolds stresses due to the interaction of instrument tilt and waves indicated that this is the dominant source of wave associated error in the uncorrected Reynolds stress calculations.

The separation distance in the along beam subtraction must be carefully chosen to balance potentially conflicting constraints. It must be small enough for the wave velocities to be highly correlated so that computing an average Reynolds stress makes 
sense over this distance. It also must be large enough that the turbulent components of the motion are largely un-correlated. A constant $1 \mathrm{~m}$ separation distance seemed to work well with our data, although in some cases it may have removed some of the larger scale turbulence causing our Reynolds stresses to be underestimated. In general we expect that an optimal separation distance would depend on the turbulence characteristics of the specific flow. For example, in a strongly wall bounded flow (e.g., a tidal channel) a separation distance that varies with the distance from the boundary may be appropriate to capture the well known scaling of the largest turbulent eddies.

We determined the wave orbital velocity scaling factor by fitting a wave velocity variance profile based on linear wave theory to the observed velocity variances over the water column, based on the assumption that most of the velocity variance in this relatively low turbulent energy system was due to wave orbital velocities. During periods of high wind and waves, the near surface velocity variances developed a distinctly different character than the remainder of the water column, presumably due to the effects of breaking waves. The height to which the linear wave theory based variance profiles fit the observed velocity variances established a diagnostic for separating this near surface region from the remainder of the water column. Stresses beneath this level were quite consistent with the background flow and shear. Above this level the magnitude remained consistent with a surface drag law, although the direction was noisy and somewhat inconsistent with the wind direction.

Overall, Reynolds stress values decreased as the gradient Richardson number increased up to a gradient Richardson number of approximately one. Above this, Reynolds stresses were independent of Richardson number and essentially at the instrument noise floor. 
While this effort may have pushed the capabilities of the ADCP instrument beyond its originally intended purposes, we have been encouraged by its robustness in providing information on Reynolds stress during this and several other similar deployments in a wind wave dominated system. 


\section{References}

Agrawal YC, and Aubrey DG, (1992) Velocity observations above a rippled bed using laser Doppler Velocimetry. J. Geophys. Res., 97, 20249-20259

Brown J, Colling A, Park D, Phillips J, Rothery D, and Wright J, (1989) Waves, Tides and Shallow-Water Processes. Pergamon Press, Oxford: 187 pp.

Dean RG, Dalrymple RA, (2002) Coastal processes with engineering applications. Cambridge University Press.

Large WG, and Pond S, (1981) Open ocean momentum flux measurements in moderate to strong winds. J. Phys. Oceanogr. 11, 324-336

Lu Y, and Lueck RG, (1999) Using a broadband ADCP in a tidal channel: part 1; Mean flow and shear. J. Atmos. Oceanic Technol. 16, No. 11: 1556-1567

Lu Y, and Lueck RG, (1999) Using a broadband ADCP in a tidal channel: part 2. turbulence. J. Atmos. Oceanic Technol. 16, No. 11: 1568-1579

Luettich RA Jr, McNinch JE, Paerl HW, Peterson CH, Wells JT, Alperin M, Martens CS, and Pinckney JL, (2000) Neuse River Estuary modeling and monitoring project stage 1: hydrography and circulation, water column nutrients and productivity, sedimentary processes and benthic-pelagic coupling, Report UNC-WRRI-2000-325B, Water Resources Research Institute of the University of North Carolina, Raleigh, NC, 172p.

Luettich RA Jr, Carr SD, Reynold-Fleming JV, Fulcher CW, and McNinch JE, (2002) Semi-diurnal seiching in a shallow, micro-tidal lagoonal estuary, Continental Shelf Research 22 No. 11-13: 1669-1681

Reynolds-Fleming JV, Fleming JG, and Luettich RA, (2002) Portable, autonomous vertical profiler for estuarine applications, Estuaries, 25(1):142-147.

Reynolds-Fleming JV, and Luettich RA Jr., (2004) Wind-driven lateral variability in the upper Neuse River Estuary, Estuarine Coastal and Shelf Science, 60:395-407.

Rippeth TP, Simpson JH, and Williams E, (2003) Measurement of the Rates of Production and Dissipation of Turbulent Kinetic Energy in an Energetic Tidal Flow: Red Wharf Bay Revisited. Journal of Physical Oceanography 33:1889-1901

Stacey MT, Monismith SG, and Burau JR, (1999) Measurements of Reynolds stress profiles in unstratified tidal flow. Journal of Geophysical Research 104 No C5: 10,93310,949

Trowbridge JH, (1998) On a technique for measurement of turbulent shear stress in the presence of surface waves. J. Atmos. Oceanic Technol. 15: 290-298

Williams E, and Simpson JH, (2004) Uncertainties in Estimates of Reynolds Stress and TKE Production Rate Using the ADCP Variance Method. J. Atmos. Oceanic Technol. 21:347-357 